\title{
Archéopages
}

Archéopages

Archéologie et société

42 | 04-07/2015

Construire en terre crue

\section{La terre crue, matériau universel}

\section{Claire-Anne de Chazelles, Hubert Guillaud et Catherine Chauveau}

\section{OpenEdition \\ Journals}

Édition électronique

URL : https://journals.openedition.org/archeopages/1317

DOI : 10.4000/archeopages.1317

ISSN : 2269-9872

\section{Éditeur}

INRAP - Institut national de recherches archéologiques préventives

\section{Édition imprimée}

Date de publication : 1 mars 2016

Pagination : 116-121

ISSN : 1622-8545

\section{Référence électronique}

Claire-Anne de Chazelles, Hubert Guillaud et Catherine Chauveau, «La terre crue, matériau universel », Archéopages [En ligne], 42 | 04-07/2015, mis en ligne le 01 juillet 2017, consulté le 02 juin 2021. URL http://journals.openedition.org/archeopages/1317; DOI : https://doi.org/10.4000/archeopages.1317 


\title{
La terre crue, matériau universel
}

\author{
Disponible, plastique, recyclable, la terre convient pour bâtir toutes \\ formes et dimensions de constructions. Mais qu'en est-il de l'évolution \\ de ces techniques constructives et de l'intérêt que les populations anciennes \\ comme actuelles lui portent de par le monde?
}

\section{6

\section{Hubert Guillaud}

spécialiste des architectures de terre, enseigne à l'École Nationale Supérieure d'Architecture de Grenoble et co-dirige l'unité de recherche AE\&CC (Laboratoires CRAterre et Cultures constructives). Il a publié notamment : avec H. Houben, Traité de construction en terre, Editions Parenthèses, 360 p., 2006 ; «Évolution de la culture constructive du pisé », in Actes des $2^{\grave{e}}$ Échanges transdisciplinaires sur les constructions en terre crue (28-29 mai 2005, Villefontaine), Éditions de l'Espérou, Montpellier, 2007, p. 277-310 ; « De traces en repères choisis : éloge de la brique crue », in Actes des $3^{\grave{e}}$ Échanges transdisciplinaires sur les constructions en terre crue. La brique crue, (16-17 mai 2008, Toulouse), Éditions de l'Espérou, Montpellier, 500 p., 2010.

\section{Claire-Anne de Chazelles}

est archéologue au CNRS, dans l'équipe «Archéologie des Sociétés Méditerranéennes ». Ses recherches concernent les techniques de construction des périodes préhistoriques et historiques, et plus spécialement les procédés utilisant la terre crue, sur le pourtour occidental de la Méditerranée. Elle a publié, notamment : avec A. Klein (dir.), Échanges transdisciplinaires sur les constructions en terre crue, Actes de la table-ronde de Montpellier, 17-18 nov. 2001, Montpellier, Éditions de l'Espérou, 460 p, 2003 ; avec A. Burens-Carroza et L. Carroza, « Les maisons en Languedoc de la fin du Néolithique à la fin de l'âge du Fer », in Architectures protohistoriques en Europe occidentale du Néolithique final à l'âge du Fer, Actes du $127^{\circ}$ congrès national des sociétés historiques et scientifiques, Nancy, 15-20 avril 2002, Paris, éditions du CTHS, p. 429-461, 2005; avec H. Guillaud et A. Klein (dir.), Les Constructions en terre massive pisé et bauge. Actes des $2^{\grave{e}}$ Echanges transdisciplinaires sur les constructions en terre crue, Villefontaine, 28-29 mai 2005, Montpellier, Éditions de l'Espérou, 328 p., 2007 ; Les Maisons en terre de la Gaule méridionale, Montagnac, Éditions Monique Mergoil, 1997, Monographies Instrumentum 2, 230 p. ; avec J.-C. Roux, « La construction en terre crue en France méditerranéenne », in Archéologie des rivages méditerranéens : 50 ans de recherche, Actes du colloque d'Arles 2829-30 octobre 2009, Paris, Errance, p. 343-347, 2010; «Quelques pistes de recherche sur la construction en terre crue et l'emploi des terres cuites architecturales pendant l'Âge du fer dans le bassin occidental de la Méditerranée », in Grecs et indigènes de la Catalogne à la mer Noire, Actes des rencontres du programme européen Ramses2, 20062008, Paris-Aix-en-Provence, ErranceCentre Camille-Jullian, coll. Bibliothèque d'archéologie méditerranéenne et africaine $\mathrm{n}^{\circ}$ 3, p. 301-318, 2010 ; avec A. Klein et N. Pousthomis (dir.), Les Cultures constructives de la brique crue, $3^{e}$ Échanges transdisciplinaires sur les constructions en terre crue, Actes du colloque international de Toulouse, 16 et 17 mai 2008, Montpellier, Éditions de l'Espérou, 502 p., 2011. 

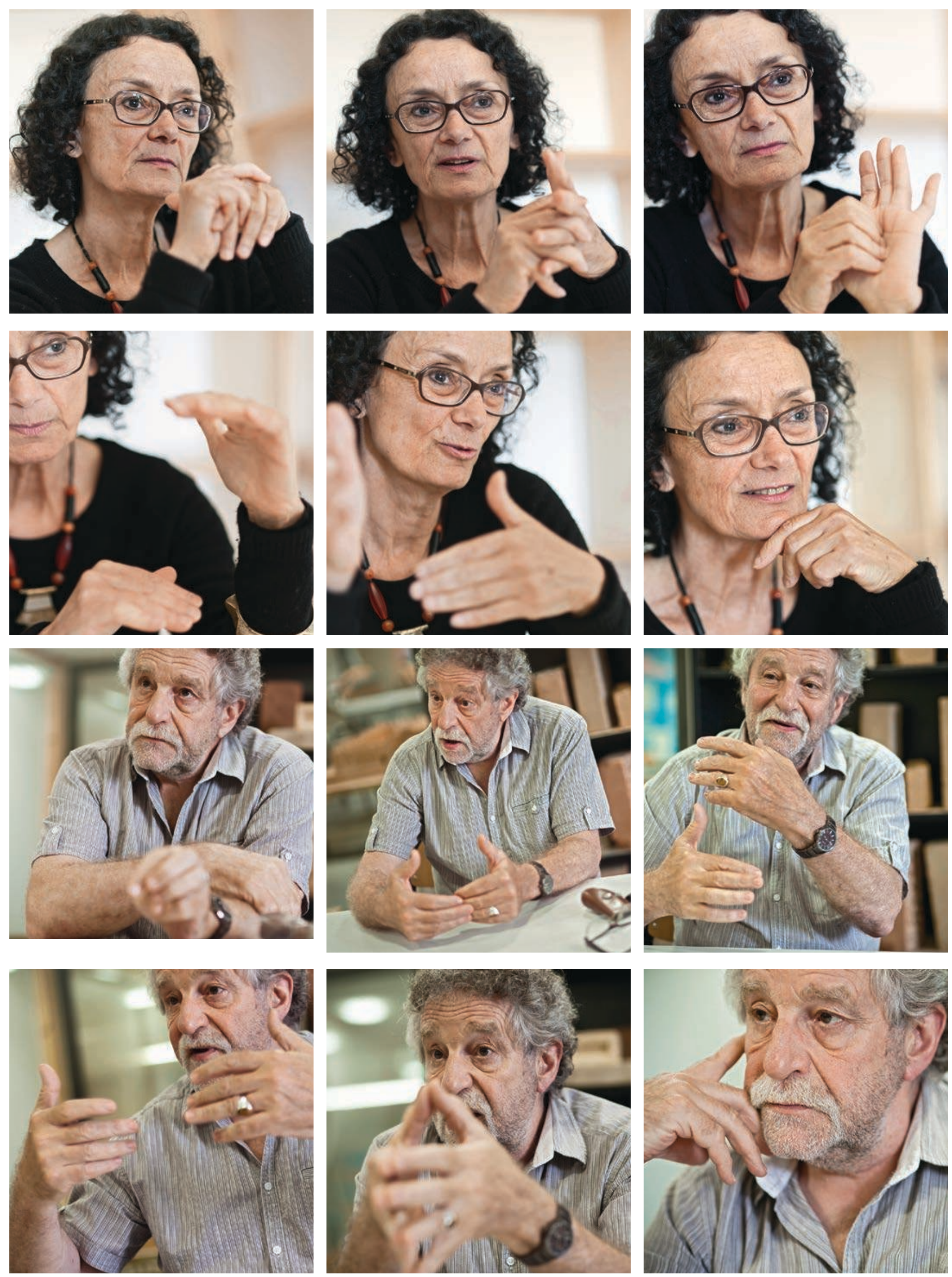

$\Xi$ 
Hubert Guillaud Lorsque j'ai commencé à m'intéresser à la construction en terre, c'était en lien avec les questions d'énergie, à la suite de la crise des années 1970. Les seules alternatives qui se développaient alors étaient, aux Etats-Unis, celles liées à l'architecture solaire passive (passive solar energy) autour d'un renouveau de la construction en briques d'adobe. Avec l'équipe de CRAterre', en tant qu'architectes, nous avons testé sur la terre l'ensemble des propriétés à requérir d'un matériau de construction (physiques, mécaniques, thermiques, de résistance à la compression...) et nous nous sommes aperçus que la terre possède de forts atouts ; par exemple, celui de la régulation de l'hygrothermie, primordial pour le confort d'un habitat et la facilité et le faible coût de la construction en terre, qui a permis tout autant la construction à léchelle domestique que celle, quasi industrielle, des premières grandes agglomérations orientales. Dans notre approche, qu'on pourrait dire d'ingénierie, on a constaté que ce sont des systèmes constructifs adaptés à l'environnement qui pallient la fragilité de la terre relative à l'humidité. La solution réside dans la combinaison d'un bon soubassement avec un bon débord de toiture et avec un renforcement des ouvertures, plus exposées au système de la goutte d'eau, ainsi que des angles de murs exposés aux vents et pluie dominants. Plus tardivement, les procédés de compression et de stabilisation comme le pisé ${ }^{2}$ et le $\mathrm{BTC}^{\mathbf{3}}$ ont rendu les modules de terre crue plus résistants que certaines briques cuites et pierres tendres. Mais il suffit de respecter les systèmes constructifs protecteurs pour pouvoir construire en terre partout.

Claire-Anne de Chazelles Oui, les archéologues font le même constat. Une fois prises les précautions architecturales dont vous parlez, on peut construire en terre même dans des régions très humides (en Normandie, par exemple, on trouve des maisons de terre qui ont plusieurs siècles) et la terre peut-être un matériau durable (dans le sud de la France, des maisons médiévales de six cents à sept cents ans, sont toujours debout sur trois niveaux, et habitées). Donc, bien protégée, la terre est indestructible. Or les archéologues avaient de faux a priori là-dessus, pour deux raisons principalement : l'impression que ce qui est archéologique ne dure pas et le constat que les protohistoriques reconstruisaient leur maison tous les 25-30 ans, comme on l'a observé, par exemple sur les sites de Martigues et de Lattes, où se sont formés de véritables tells. Où en est d'ailleurs la recherche sur

5. Deux exemples en bauge : les cases obus du Cameroun, rondes, montées comme au colombin, avec des murs minces pour ne pas accumuler la chaleur; les maisons du Yemen, angulaires, comportant jusqu'à huit étages mais sous ces climats, les murs sont plus épais et reposent sur des fondations de pierre. la consolidation et la présentation des vestiges en terre quand ils sont conservés sur plusieurs mètres de haut?

HG Suivant les conditions climatiques et les mesures de protection initiales, les constructions sont très vite dégradées, soit parce que les procédés choisis ont des effets induits négatifs ${ }^{4}$ soit parce qu'aucun suivi ni entretien ne sont prévus. Plusieurs expérimentations ont été faites : les enduits dits « sacrificiels »; les protections géotextiles recouvert de mortier terreux qui ont l'avantage d'être réversibles ; le drainage permanent aux abords des structures. La reconstruction en partie s'impose souvent à cause d'une fragilité structurelle : un pan de mur exposé à effondrement, par exemple, est reconsolidé par réintégration de même matériau dans les lacunes. Aujourd'hui, nous optons pour des interventions a minima précédées d'analyse a maxima des structures, afin de s'adapter plus précisément aux caractéristiques du site et de ne pas nuire à son authenticité. Et nous devons réussir cela alors que les financements de la conservation sont généralement très sous-évalués !

C-AC Pour revenir aux bons principes de construction, nous les constatons par nos expérimentations. Une des maisons expérimentales de Lattes s'est écroulée pendant l'hiver et on a bien compris que c'était un problème de couverture. Si on lui avait fait une toiture végétale pentue, on n'aurait pas eu ce problème. Mais j'ai opté pour un toit plat, en accord avec les propositions de Patrice Arcelin et de Jean Chausserie-Laprée, qui, d'après les fragments épais de terre avec empreintes de roseaux retrouvés sur le site de Martigues et des comparaisons ethnologiques avec l'Afrique du Nord avaient posé cette hypothèse. Il y a, à Lattes, diverses pièces de terre cuite avec une pâte proche de celle des amphores marseillaises dont je pense qu'elles sont des gargouilles et des déversoirs pour l'eau de pluie; ce qui colle plutôt avec l'option toits plats. Dans un urbanisme aussi compact que celui de Lattes ou de celui de Martigues, les toits plats sont une solution plus pratique, pour juxtaposer les maisons, notamment. Les ruelles étant très étroites, j'ai du mal à imaginer des toits de chaumes à versants et les toits plats m'ont paru une bonne solution.

HG Quelque soit le matériau, le problème des constructeurs et de répartir les forces, d'éviter que les murs ne s'ouvrent ou ne s'affaissent. L'évolution du schéma des bâtiments est toujours la même partout: on commence par les maisons rondes, et on tend vers la maison quadrangulaire, ou pas ! La forme ronde est une forme parfaite car toutes les forces s'équilibrent et la charge de la toiture est parfaitement répartie. Les questions de répartition des forces sont plus complexes dans les maisons quadrangulaires. L'avantage du petit élément qu'est la brique de terre crue est qu'on peut l'appareiller pour obtenir toute épaisseur de murs et surtout des angles droits rigoureux. La brique donne beaucoup de flexibilité d'emploi et conduit plutôt à formes orthogonales que courbes. L'autre question que peuvent se poser les bâtisseurs est lintérêt de lélévation. Dans le monde occidental, que l'on construise en brique crue, en bauge, en pisé, lélévation n'excède pas trois étages maximum. Les techniques de terre permettent cependant de monter plus haut ${ }^{5}$. Les variabilités des logiques constructives résultent à la fois de l'usage des matériaux à disposition, évidemment, 


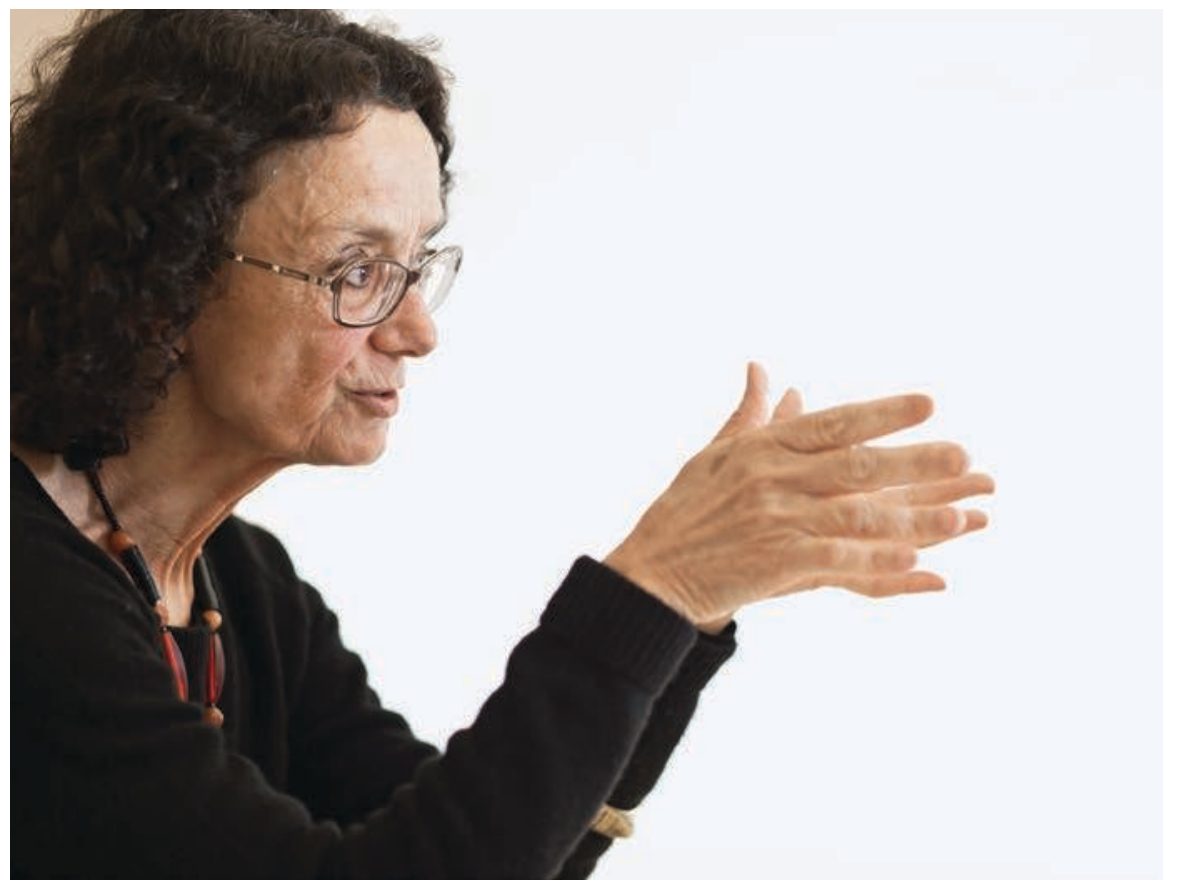

\title{
Le principe même de la construction en terre, c'est l'adaptation au milieu.
}

\author{
Claire-Anne de Chazelles
}

6. Apparue au ProcheOrient au VIIe millénaire, la construction en briques crues n'est adoptée, jusqu'aux années 4000 avant notre ère qu'en Méditerranée orientale, en Grèce et en Égypte, et n'es connue en Méditerranée occidentale qu'à partir des colonisations phénicienne et grecque. souvent lié à une intelligence qui permet d'en tirer le meilleur parti mais aussi d'expérimentations répétées jusqu'à ce que les procédés techniques aboutissent à des modèles culturellement marquants. Ces logiques de construction s'imposent de fait par une intelligence mémorielle de l'art de construire transmis de génération en génération. Pour les époques modernes, en France, on constate bien ces particularités régionales, dans les choix des techniques et des associations de matériaux : le pisé (particulièrement efficace en zones tempérées à froides pour amortir les différences entre les températures extérieures et intérieures), la brique crue, la bauge en association avec la pierre, le bois, le mortier de chaux, la brique cuite, des fibres végétales pour renforcer les endroits plus fragiles, épaissir les murs, améliorer la tenue de la terre... Partout en France, le torchis a donné, dans sa forme la plus élaborée et extrêmement flexible, le colombage, à bois courts ou à bois longs suivant les influences anglo-saxonnes ou celles plutôt méridionales. Quant aux combinaisons fréquentes de briques crues et cuites, elles sont un bon compromis entre l'avantage des premières, économiques et pratiques, et celui des secondes, plus solides et durables mais plus chères. La diversité des solutions est grande mais chaque technique renvoie à des typologies finalement peu différentes. Et les techniques complexes (comme le pisé) ont dû être mises au point après plusieurs expérimentations. Il est notable que ce qui s'apparente à la technique du pisé, quel que soit le nom qu'on lui donne, est très homogène d'un continent à l'autre actuellement. Ce qui varie, ce sont les procédés (types de coffrages ou de fouloirs) et les organisations et spécialisations du travail.
C-AC Oui, on sétonne que des techniques si anciennes soient à ce point figées. Les techniques constructives en terre sont assez intuitives et se sont généralisées y compris dans les régions où il y a de la pierre et du bois. Le fait que la bauge existe depuis des millénaires sur tous les continents est sans doute une des explications de sa grande diversité, dans les formes des éléments modelés à la main et les façons de les assembler, mais le procédé en lui-même n'évolue pas. Le bâti en brique crue moulée est bien moins variable, dès ses débuts et sur le long terme. Les dimensions différent assez peu et on a même une constante mondiale d'une épaisseur comprise entre 7 et $9 \mathrm{~cm}$. Il s'agit, en effet, de trouver un équilibre entre des dimensions suffisamment grandes pour monter rapidement des murs et celles permettant de manipuler les modules sans trop d'effort ; on trouve cependant parfois des briques de très grandes dimensions, disposées à plat ou de champ, qui ne pouvaient être manipulées qu'à deux. Bien que la brique crue représente une réelle rationalisation de la construction, la diffusion de cette technique est relativement lente ${ }^{\mathbf{6}}$. En prenant l'exemple de Lattes, au début de l'occupation, début du $\mathrm{V}^{\mathrm{e}} \mathrm{s}$. siècle avant notre ère, les constructions sont essentiellement en bauge, alors que la brique crue est déjà bien utilisée sur le pourtour méditerranéen ; et ce n'est qu'environ un siècle plus tard que l'emploi de la brique crue y est généralisé. Serait-ce dû à des résistances culturelles, comme l'échec ou le succès de certains procédés? Le pisé, particulièrement efficace, n'a pas été utilisé partout sans que la raison soit liée de façon évidente à des facteurs climatiques (notamment lorsque le sous-sol est humide en permanence). Il y a des régions qui, indépendamment de la ressource, 
optent résolument pour la terre. C'est le cas du site protohistorique du Marduel, habitat perché au dessus de la vallée du Gardon. En plein un massif calcaire, les pierres ne servent que pour les soubassements et les murs sont en briques crues ; les habitants ont préféré produire les briques au bord de la rivière et les remonter sur l'oppidum. C'est un exemple parmi d'autres, de choix des moyens locaux. L'image dominante de la construction aux $\mathrm{V}^{\mathrm{e}}$, IV $^{\mathrm{e}}, \mathrm{III}^{\mathrm{e}}$ siècles avant notre ère, est la maison en briques donc certains font des maisons en briques alors que la pierre est le matériau local. En général, quand même, on constate une adaptation au milieu et aux ressources avec un schéma de construction identique. Par exemple, au Néolithique, dans le sud de la France, on a un même type de maisons, sans que cette forme soit « dictée » par le matériau : elles sont en pierre dans les garigues, et en terre dans la plaine de Lattes, où il n'y a pas un caillou et pas un arbre. HG Le choix du matériau n'apparaît donc pas forcément comme un marqueur de distinction sociale?

C-AC Lorsque tout le monde construit en terre, ce ne peut être un signe de pauvreté. D'économie, de commodité, peut-être. À l'époque romaine, dans la mesure où tout est dissimulé sous des enduits, de très belles maisons ont des murs en terre. Dans le sud de la France, on a construit en terre durant tout le Moyen Âge mais ensuite, on a l'impression que cela se raréfie, au moins en contexte urbain. La construction en terre perdure à la campagne mais avec des variabilités qu'on ne comprend pas encore. Par exemple, en Provence, la construction en pisé existe jusqu'au $\mathrm{xx}^{\mathrm{e}}$ siècle, ${ }^{\mathrm{e}}$ siècle alors qu'en Languedoc, on a l'impression que la terre est alors soudain et totalement abandonnée au profit de la pierre. Les gens utilisent la terre quand c'est le matériau disponible et souvent, terre et pierres sont sous les enduits ; à part les entourages d'ouvertures en pierre, bien visibles et qui peuvent être une volonté d'affirmer un statut plus élevé. C'est la même ostentation durant l'époque moderne en Provence, lorsque le bâtiment d'habitation d'un mas présente une façade avant de pierres, ou que tout le rez-de-chaussée a un appareillage de pierres visible. Mais dans ce cas, il peut s'agir aussi d'une protection contre des aléas naturels : dans la vallée de la Durance, on sait par des textes que des quartiers entiers se sont écroulés suite à une crue. Dans d'autres aires géographiques méditerranéennes que je connais, tout le monde construit en terre. Sur le site où je travaille en ce moment au Maroc ${ }^{7}$, pour la période préromaine, il n'y a absolument pas de pierres. Ce n'est donc pas comme cela que se marque la différenciation sociale. En général, elle se traduit par de plus grandes dimensions, des finitions plus soignées, une ornementation abondante, des emprunts à des cultures prestigieuses... C'est la porosité entre cultures qui fait évoluer les mentalités. Lorsque vous initiez des équipes locales, par exemple en Afrique subsaharienne où l'on a toujours construit en bauge, à des techniques comme le pisé ou le BTC, est-ce que ces gens se l'approprient ou bien sont-ils réticents? Est-ce que cela modifie leur technique constructive par la suite?

HG La construction moderne industrielle a laminé les cultures constructives dans la plupart des pays où nous allons et a imposé une vision de l'architecture moderne fondée sur le béton, sur l'acier, qui conditionne les aspirations de la population. Le matériau terre leur est présenté comme pauvre et arriéré et les renvoie à une image négative d'eux-mêmes. Ces pays ont souvent produit des parpaings ou des briques cuites, à grand renfort de destruction environnementale, et les projets n'aboutissaient souvent pas, faute de maîtrise et d'adaptation technique. Pour nous, il ne s'agit donc pas de faire du transfert de technologies mais de répondre à des attentes de la population : accéder à un habitat de meilleure qualité et vivre de l'économie de la construction. Le travail avec les populations locales passe par des démonstrations techniques, par un travail en réseau ${ }^{\mathbf{8}}$ et par des projets de constructions qui aident à redonner une vision positive de l'architecture de terre, des projets en BTC d'une intelligence remarquable et d'une grande modernité formelle, comme le travail de l'architecte Francis Kere au Burkina Faso. Dans un autre contexte, notre projet en Haïti après le tremblement de terre, concernait le milieu rural et se fondait sur la tradition de la culture architecturale haïtienne mais avec des matériaux modernes de qualité. À Mayotte, où les habitats traditionnels, principalement faits de végétaux, sont mis à bas aux passages des queues de cyclone, l'enjeu était de développer des habitats en dur, dans un respect écologique?. Nous avons proposé de mobiliser des ressources locales, de la terre argileuse latéritique et de la terre d'origine pouzzolanique. En mélangeant les deux, la pouzzolane jouant le rôle de dégraissant par rapport à l'argile, on a obtenu une formule de terre de très bonne qualité pour faire du BTC; il peut d'ailleurs être stabilisé à 3 ou $4 \%$ d'un peu de ciment, ou de chaux, ce qui le rend résistant à l'humidité. Ces exemples de projets ont été suffisamment convaincants pour redéployer, ou déployer, la filière terre dans des contextes où les populations avaient assimilé une vision péjorative de ce matériau et perdu en partie leur savoir-faire. Et tout cela en préservant un environnement ! Car l'extraction de la terre peut se faire sans dommage. La technique de base dite « en mottes de gazon », pratiquée dans l'Europe médiévale et dans l'Amérique latine actuelle, par exemple, est un décapage superficiel de terre végétale, par rotation, de façon à ce que l'humus se régénère. Évidemment, si on creuse sur de grande profondeur, on détruit et on stérilise le sol, ce qu'il faut éviter. Mais il devrait être possible d'exploiter des types de veines souterraines, en carrière, sans dégradation du milieu. Existe-t-il des traces archéologiques d'exploitation intensive de terre?

C-AC Pour l'instant, on n'en connaît pas. En ce qui concerne mes aires de recherche, pour Lattes, 


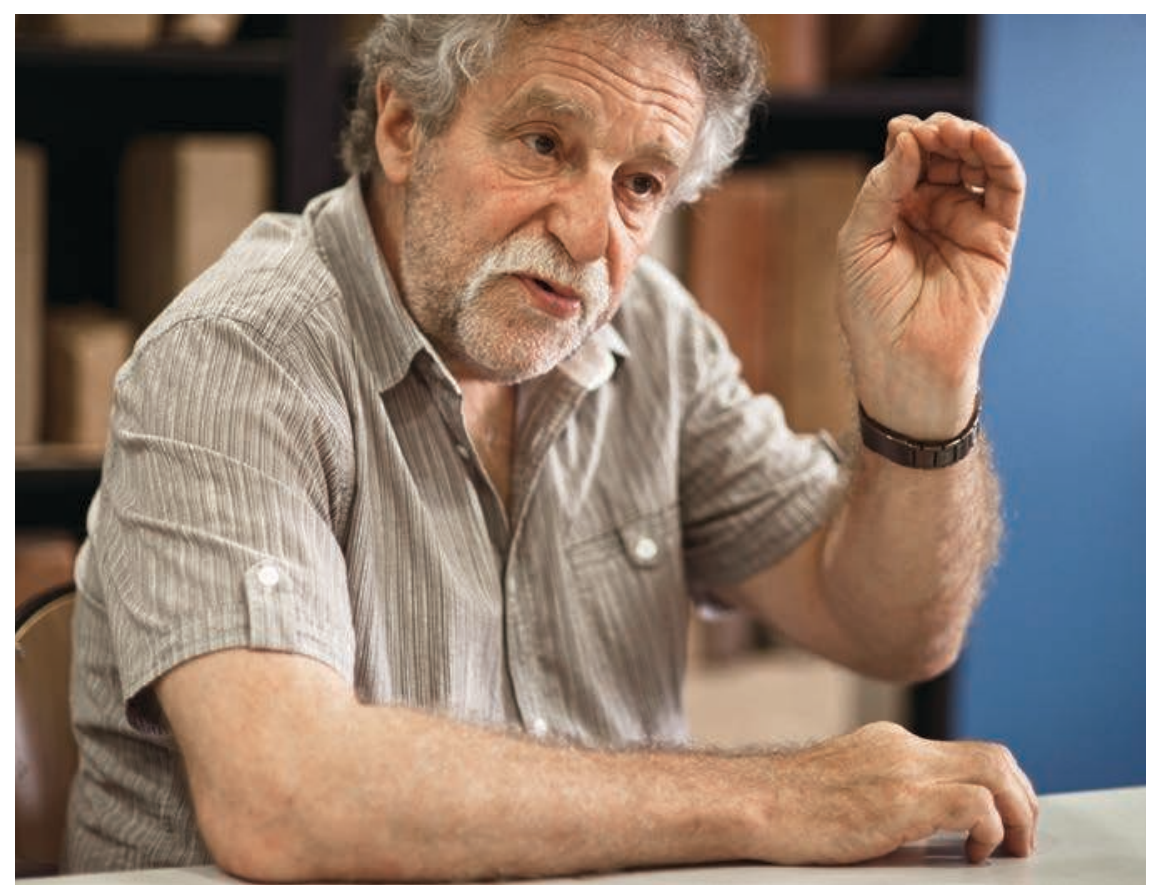

Le matériau terre souffre d'une vision désuète et péjorative alors qu'il présente de très nombreux atouts architecturaux et environnementaux.

Hubert Guillaud les berges du Lez ont été tellement remaniées qu'on a peu de chance de les trouver; dans la région où je travaille au Maroc ${ }^{\mathbf{1 0}}$, les gens font encore des briques et les traces des petites carrières sur les bords du oued sont visibles. Ce ne sont que des emplacements espacés car la terre n'a qu'un usage domestique limité. Mais les travaux d'ampleur urbains et défensifs d'Orient et d'Occident, de la Protohistoire au Moyen Âge, ont dû mobiliser énormément de matériau. Cependant, le grand avantage de la terre est d'être réutilisable. Le matériau disponible peut donc être les bâtiments eux-mêmes. Au v viècle avant notre ère, les gens de Lattes sont allés choisir de bonnes terres, près du Lez. Puis, au fur et à mesure des démolitions, ils ont récupéré les matériaux et les ont mélangés. Une maison en terre devait être constamment entretenue et les traces de ces réparations sont fréquentes (sols rechapés, murs réenduits, rebouchés...). Cela pourrait expliquer que la reconstruction ait été préférée à un entretien sur le long terme, et même que la terre soit abandonnée pour la pierre. Encore fallait-il avoir les moyens ! La reconstruction est souvent basée sur des remplois et des récupérations. On s'en rend compte notamment lors des études de murs en briques crues : certaines ont des faces cuites et sont sans aucun doute des briques cuites lors d'un incendie et réutilisées dans une nouvelle construction. La terre de démolition se recycle indéfiniment : il suffit de remettre de la paille fraîche et de l'eau. D'ailleurs, jusqu'à ces dernières années, les archéologues pensaient que le torchis prédominait, car il se distingue bien en fouille ${ }^{\mathbf{1 1}}$. Il est plus difficile de repérer le pisé, mais il était sans doute plus répandu qu'on ne l'imagine. Le choix de la technique a quand même un lien avec la nature de la terre. Dans nos expérimentations à Lattes, on a pris le parti d'utiliser la même terre locale pour tout, mais elle se révèle trop peu argileuse. Elle reste tout à fait efficace pour les briques crues et pour les enduits mais pas pour faire du pisé. Il semble donc que toutes les terres ne conviennent pas aussi bien.

HG A priori, on peut se servir de tout type de terre parce que si la terre naturelle ou de récupération n'est pas convenable, on va pouvoir la corriger en y ajoutant d'autres éléments minéraux ou végétaux. Pour la brique, pour la bauge, il n'y a pas de problème, en tous cas : toute terre se travaille. Et certains choix semblent déterminés moins par la nature de la terre que par sa teinte, dans la recherche d'un aspect esthétique.

C-AC C'est très manifeste. Le corps d'enduit est fait avec une terre banale, et la finition avec une argile fine, d'une couleur décorative. À Lattes, on retrouve des fragments d'enduits avec plusieurs couches de couleurs différentes comme si on avait changé la peinture des murs régulièrement : $a u \mathrm{II}^{\mathrm{e}}$ siècle, murs et sols sont de divers tons d'ocre jaune foncé ; à la période antérieure, ils sont dans des tons clairs (vert pâle, beige, blanc). Pour bien comprendre la spécificité des enduits, on met en place un programme de recherche sur l'analyse des matières organiques mêlés à la terre. On sait que les Romains mélangeaient la terre avec de la caséine ou avec le résidu de pressage des olives, corps gras qui rendent les matériaux plus ductiles et renforcent l'étanchéité. Ils s'en servaient pour enduire les greniers notamment; peut-être aussi que cela éloigne les insectes, les rats, les vers... Les recherches sur l'identification et l'utilisation de la terre dans l'architecture n'en sont quà leurs débuts ! 11. Notamment par les empreintes de végétaux dans la terre cuite par incendie. 